\title{
DESCRIPTIVE COLLINEATIONS IN SPACES OF $K$-SPREADS
}

\author{
BY \\ BUCHIN SU
}

1. Introduction. In a paper of a few years ago( $\left.{ }^{1}\right)$, Douglas has developed the geometry and tensor analysis of the space of $K$-spreads. The configuration considered in this space is formed by a system of $K$-dimensional analytic manifolds immersed in an $N$-dimensional space and distributed according to an analytic law so that $K+1$ points in general position determine uniquely a $K$-dimensional manifold containing these points. The partial differential equations of the $K$-spreads may be written in the form given by Douglas (except for the sign of the $H$ functions):

$$
\frac{\partial^{2} x^{i}}{\partial u^{\alpha} \partial u^{\beta}}+H_{\alpha \beta}^{i}(x, p)=0 \quad\left(p_{\alpha}^{j}=\frac{\partial x^{j}}{\partial u^{\alpha}}\right),
$$

where $x^{i}(i=1,2, \cdots, N)$ denote coordinates of a point and $u^{\alpha}(\alpha=1,2$, $\cdots, K)$ parameters on each $K$-spread. The functions $H_{\alpha \beta}^{d}$ obey the condition of the generalized homogeneity property

$$
H_{\alpha \beta}^{i}(x, q)=A_{\alpha}^{\lambda} A_{\beta}^{\mu} H_{\lambda \mu}^{i}(x, p),
$$

when the parameters $u^{\alpha}$ are subjected to by linear transformations of the form

$$
u^{\alpha}=A_{\lambda}^{\alpha} v^{\lambda}+C^{\alpha}
$$

so that

$$
\partial x^{i} / \partial v^{\alpha}=A_{\alpha}^{\lambda} \partial x^{i} / \partial u^{\lambda}, \text { or } q_{\alpha}^{i}=A_{\alpha}^{\lambda} p_{\lambda}^{i} .
$$

Of the various geometries of $K$-spreads treated by Douglas, E. T. Davies( $\left.{ }^{2}\right)$ has recently enriched the affine geometry by introducing isomorphic transformations which may be seen as affine collineations of the space, as equations (1.1) are determined uniquely to within (1.3). If, on the contrary, we make in (1.1) an arbitrary transformation of parameters:

$$
u^{\alpha}=u^{\alpha}(v ; a) \text {, inversely } v^{\alpha}=v^{\alpha}(u ; a),
$$

containing a set of constants $a^{1}, a^{2}, \cdots, a^{R}, R=(N-K)(K+1)$, then the equations of the space of $K$-spreads referred to the new parameters $v$ become

Presented to the Society, December 29, 1946; received by the editors July 11, 1946.

(1) J. Douglas, Systems of $K$-dimensional manifolds in an $N$-dimensional space, Math. Arin. vol. 105 (1931) pp. 707-733.

(2) E. T. Davies, On the isomorphic transformations of a space of $K$-spreads, J. London Math. Soc. vol. 18 (1943) pp. 100-107. 


$$
\frac{\partial^{2} x^{i}}{\partial v^{\alpha} \partial v^{\beta}}+H_{\alpha \beta}^{i}(x, q)=q_{\lambda}^{i} G_{\alpha \beta}^{\lambda}(x, q),
$$

where the system $G_{\alpha \beta}^{\lambda}$ is homogeneous of the character represented by its indices. It is desirable to give a corresponding theory of isomorphic transformations for the descriptive geometry of $K$-spreads. In this paper, we shall study descriptive collineations as a generalization of projective collineations in the general geometry of paths considered by Knebelman $\left({ }^{3}\right)$. In $\$ 2$, we find the equations of the space of $K$-spreads in determinant form which is unchanged by (1.5), and therefrom derive the equations expressing the conditions for an infinitesimal descriptive collineation which the space may admit. $\$ 3$ deals with the conditions of integrability of these equations. As in the case of affine geometry the notion of the Lie derivative is utilized in reducing the number of these conditions and derived equations. Immediate consequences are obtained in $\S 4$. The paper concludes with a study of the group property of descriptive collineations $\left({ }^{4}\right)$.

2. The equations of the space of $K$-spreads and descriptive collineations. Let $\bar{x}^{i}=\phi^{i}(x)$ be a point transformation which carries the point $\left(x^{1}, \cdots, x^{N}\right)$ into the point with coordinates $\left(\bar{x}^{1}, \cdots, \bar{x}^{N}\right)$. Let $x^{i}=f^{i}\left(u^{1}, u^{2}, \cdots, u^{K}\right)$ be the finite equations of a $K$-spread $M$, the parameters $u^{\alpha}$ being affine, that is, the equations satisfying (1.1) or

$$
\frac{\partial^{2} x^{i}}{\partial u^{\alpha} \partial u^{\beta}}+\Gamma_{j k}^{i}(x, p) p_{\alpha}^{i} p_{\beta}^{k}=0, \quad\left(\Gamma_{j k}^{i}=\Gamma_{k j}^{i}\right) .
$$

The transformation $\bar{x}^{i}=\phi^{i}(x)$ will carry $M$ into a manifold $\bar{M}$ of $K$ dimensions whose finite equations are of the form $\bar{x}^{i}=\psi^{i}\left(u^{1}, u^{2}, \cdots, u^{K}\right)$. This transformation will be called a descriptive collineation if it carries each $K$-spread $M$ into a $K$-spread $\bar{M}$.

Since the parameters on the $K$-spread are not necessarily preserved it will be convenient to take the equations of the $K$-spreads in determinant form:

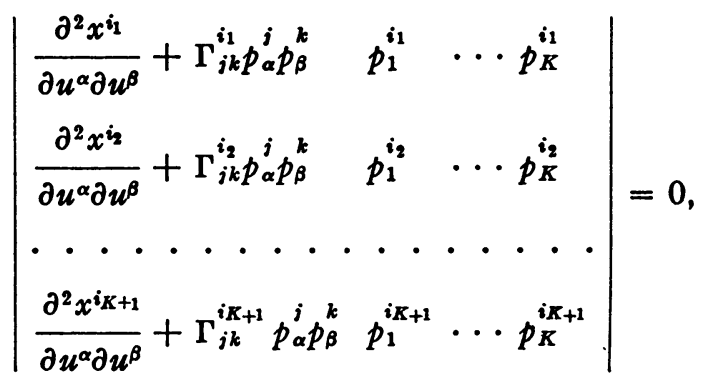

(3) M. S. Knebelman, Collineations and motions in generalized spaces, Amer. J. Math. vol. 51 (1929) pp. 527-564.

() For affine collineations see my paper: Group property of isomorphic transformations in a space of $K$-spreads, appearing soon in England. 
which are invariant under arbitrary transformations of coordinates as well as under arbitrary transformations of parameters. In (2.2), $i_{1}, i_{2}, \cdots, i_{K+1}$ denote any set of $K+1$ indices in the range $1,2, \cdots, N$.

We shall consider the problem of determining the conditions satisfied by the functions $\xi^{i}(x)$ such that the infinitesimal transformation

$$
\bar{x}^{i}=x^{i}+\xi^{i}(x) \delta t
$$

shall determine a one-parameter group of descriptive collineations, where $\xi^{i}(x)$ are the components of a contravariant vector and $\delta t$ is a differential of the parameter of the group. By differentiation we obtain from (2.3)

$$
\frac{\partial \bar{x}^{i}}{\partial u^{\alpha}}=\frac{\partial x^{i}}{\partial u^{\alpha}}+\frac{\partial \xi^{i}}{\partial x^{j}} p_{\alpha}^{j} \delta t, \quad \text { or } \quad \bar{p}_{\alpha}^{i}=p_{\alpha}^{i}+\frac{\partial \xi^{i}}{\partial x^{j}} p_{\alpha}^{j} \delta t
$$

and, with $p_{\alpha \beta}^{j}=\partial^{2} x^{j} /\left(\partial u^{\alpha} \partial u^{\beta}\right)$,

$$
\frac{\partial^{2} \bar{x}^{i}}{\partial u^{\alpha} \partial u^{\beta}}=\frac{\partial^{2} x^{i}}{\partial u^{\alpha} \partial u^{\beta}}+\left(\frac{\partial^{2} \xi^{i}}{\partial x^{j} \partial x^{k}} p_{\alpha}^{j} p_{\beta}^{k}+\frac{\partial \xi^{i}}{\partial x^{j}} p_{\alpha \beta}^{j}\right) \delta t .
$$

If (2.3) is to define an infinitesimal descriptive collineation, we must have the following transformation of the connection coefficients:

$$
\Gamma_{j k}^{i}(\bar{x}, \bar{p})=\Gamma_{j k}^{i}(\bar{x}, \bar{p}) .
$$

On writing

$$
\begin{aligned}
\Gamma_{j k}^{i}(\bar{x}, \bar{p}) & =\Gamma_{j k}^{i}(x, p)+\left(\frac{\partial \Gamma_{j k}^{i}}{\partial x^{l}} \xi^{l}+\left.\Gamma_{j k}^{i}\right|_{m} ^{0} \frac{\partial \xi^{m}}{\partial x^{n}} p_{\alpha}^{n}\right) \delta t \\
\bar{p}_{\alpha}^{j} \bar{p}_{\beta}^{k} & =p_{\alpha}^{j} p_{\beta}^{k}+\left\{p_{\alpha}^{i} \frac{\partial \xi^{k}}{\partial x^{m}} p_{\beta}^{m}+p_{\beta}^{k} \frac{\partial \xi^{i}}{\partial x^{m}} p_{\alpha}^{m}\right\} \delta t
\end{aligned}
$$

and substituting them into the corresponding equations (2.2) referred to the coordinate system $\bar{x}$, we have the following conditions satisfied by the $\xi^{i}$ :

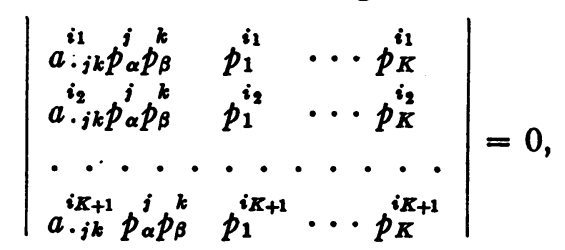

where

$$
\begin{gathered}
a_{\cdot j k}^{i} \equiv \frac{\partial^{2} \xi^{i}}{\partial x^{j} \partial x^{k}}-\Gamma_{j k}^{h} \frac{\partial \xi^{i}}{\partial x^{h}}+\Gamma_{j h}^{i} \frac{\partial \xi^{h}}{\partial x^{k}}+\Gamma_{h k}^{i} \frac{\partial \xi^{h}}{\partial x^{j}} \\
+\xi^{h} \frac{\partial \Gamma_{j k}^{i}}{\partial x^{h}}+\left.\Gamma_{j k}^{i}\right|_{m} ^{\sigma} \frac{\partial \xi^{m}}{\partial x^{h}} p_{c}^{h}
\end{gathered}
$$


and $A::\left.\right|_{m} ^{\alpha} \equiv \partial A:: /\left(\partial p_{\alpha}^{m}\right)$ for any geometric object $A::$. Here and hereafter higher powers of $\delta t$ than the first are neglected.

For the subsequent discussion it is necessary to give here the expression for the affine curvature tensor:

$$
R_{\cdot j k l}^{i}=\frac{\partial \Gamma_{j k}^{i}}{\partial x^{l}}-\frac{\partial \Gamma_{j l}^{i}}{\partial x^{k}}+\left.\Gamma_{j l}^{i}\right|_{m} ^{\tau} \Gamma_{n k}^{m} p_{\tau}^{n}-\left.\Gamma_{j k}^{i}\right|_{m} ^{r} \Gamma_{n l}^{m} p_{\tau}^{n}+\Gamma_{n l}^{i} \Gamma_{j k}^{n}-\Gamma_{n k}^{i} \Gamma_{j l}^{n}
$$

and the covariant derivation of a tensor $T^{(i)}(j)$ :

$$
\begin{aligned}
& T_{(i), k}^{(i)}=\frac{\partial T_{(i)}^{(i)}}{\partial x^{k}}-\left.T_{(i)}^{(i)}\right|_{m} ^{\sigma} p_{\sigma}^{n} \Gamma_{n k}^{m} \\
& +\sum_{\alpha=1}^{r} T^{\cdots i_{\alpha-1} h i_{\alpha+1} \cdots}{ }_{(j)} \Gamma_{h k}^{i \alpha}-\sum_{\alpha=1}^{s} T^{(i)} \cdots_{j \alpha-1} j_{\alpha+1} \ldots \Gamma_{j \alpha k}^{h} .
\end{aligned}
$$

In particular, we have the covariant derivatives of a contravariant vector $\xi^{i}(x)$ :

$$
\xi_{, j}^{i}=\partial \xi^{i} / \partial x^{j}+\Gamma_{h j}^{i} \xi^{h}
$$

and similar expressions for $\xi_{, j k} \equiv \xi_{, j, k}, \xi_{, j k l}^{q} \equiv \xi_{, j, k, l}^{\natural}$, and so on.

In virtue of these formulas the second member of (2.10) may be put in tensor form

$$
a_{\cdot j k}^{i}=\xi_{, j k}^{i}+R_{\cdot j k l}^{i} \xi^{l}+\left.\left.\Gamma_{j k}^{i}\right|_{m} ^{\sigma} \xi^{m}\right|_{r} p_{\sigma}^{r} .
$$

To find a more convenient expression for conditions (2.9) we take the equivalent ones:

$$
a_{\cdot j k}^{i} p_{\alpha}^{i} p_{\beta}^{k}=\phi_{\alpha \beta}^{\gamma} p_{\gamma}^{i},
$$

where $\phi_{\alpha \beta}^{\gamma}$ are to be determined. Partial differentiation of (2.15) with respect to $p_{\rho}^{r}$ and $p_{\sigma}^{s}$ gives

$$
\begin{aligned}
& \left.a_{\cdot j k}^{i}\right|_{r s} ^{\rho \sigma} p_{\alpha}^{j} p_{\beta}^{k}+\left.a_{., s k}^{i}\right|_{r} ^{p_{0} \delta_{\alpha} p_{\beta}^{k}}+\left.a_{\cdot{ }_{j s}}^{i}\right|_{r} ^{\rho_{\beta}} \delta_{\beta} p_{\alpha}^{j} \\
& +\left.a_{\cdot r k}^{i}\right|_{\delta} ^{\sigma} \delta_{\alpha}^{\rho} p_{\beta}^{k}+\left(\delta_{\alpha}^{\rho} \delta_{\beta}^{\sigma}+\delta_{\alpha}^{\sigma} \delta_{\beta}^{\rho}\right) a_{\cdot r \boldsymbol{r}}^{i}+\left.a_{\cdot j r}^{i}\right|_{\delta} ^{\sigma} \delta_{\beta}^{\rho} p_{\alpha}^{j} \\
& =\left.\phi_{\alpha \beta}^{\gamma}\right|_{r s} ^{\rho \sigma} p_{\gamma}^{i}+\left.\phi_{\alpha \beta}^{\sigma}\right|_{r_{s} \delta_{s}^{i}} ^{\rho}+\left.\phi_{\alpha \beta}^{\rho}\right|_{s} ^{\sigma} \delta_{r}^{i} \text {. }
\end{aligned}
$$

By contraction in (2.16) both for $\rho$ and $\alpha$ and for $\sigma$ and $\beta$ we obtain

$$
\begin{aligned}
K(K+1) a_{\cdot r s}^{i}+\left.a_{\cdot j k}^{i}\right|_{r s} ^{\alpha \beta} p_{\alpha \beta \beta}^{j} p_{\beta}^{k}+(K+1)\left(\left.a_{\cdot{ }_{j s}}^{i}\right|_{r} ^{\alpha}+\left.a_{\cdot j r}^{i}\right|_{s} ^{\alpha}\right) p_{\alpha}^{j} \\
\quad=\left.\phi_{\alpha \beta}^{\gamma}\right|_{r s} ^{\alpha \beta} p_{\gamma}^{i}+\left.\phi_{\alpha \beta}^{\beta}\right|_{r} ^{\alpha} \delta_{s}^{i}+\left.\phi_{\alpha \beta}^{\alpha}\right|_{\delta} ^{\beta} \delta_{r .}^{i} .
\end{aligned}
$$

In order to simplify this relation use is made of the original expression of $a_{\cdot j k}^{i}$, namely, (2.10), as well as the equation $\left({ }^{5}\right)$

(5) Cf. Douglas, loc. cit. p. 715, formula (5.9). 


$$
\left.\dot{p}_{\alpha}^{r} p_{\beta}^{8} \Gamma_{r a}^{i}\right|_{j k} ^{\alpha \beta}+(K+1) p_{p}^{a}\left(\left.\Gamma_{j a}^{i}\right|_{k} ^{p}+\left.\Gamma_{a k}^{i}\right|_{j} ^{p}\right)=0 .
$$

An easy calculation suffices to show that

$$
\left.a_{\cdot j k}^{i}\right|_{r s} ^{\alpha \beta} p_{\alpha}^{j} p_{\beta}^{k}+(K+1)\left(\left.a_{\cdot r i}^{i}\right|_{s} ^{\alpha}+\left.a_{\cdot j s}^{i}\right|_{r} ^{\alpha}\right) p_{\alpha}^{j}=0,
$$

so that (2.17) becomes

$$
a_{. r s}^{i}=\left.\phi_{\alpha \beta}^{\gamma}\right|_{r s} ^{\alpha \beta} p_{\gamma}^{i}+\left.\phi_{\alpha \beta}^{\beta}\right|_{r} ^{\alpha} \delta_{s}^{i}+\left.\phi_{\alpha \beta}^{\alpha}\right|_{\delta} ^{\beta} \delta_{r}^{i},
$$

the functions $\phi$ in (2.17) being now replaced by $K(K+1) \phi$.

Regarding (2.10), again we have

$$
\begin{aligned}
\frac{\partial^{2} \xi^{i}}{\partial x^{j} \partial x^{k}}-\Gamma_{j k}^{a} \frac{\partial \xi^{i}}{\partial x^{a}}+\Gamma_{j a}^{i} \frac{\partial \xi^{a}}{\partial x^{k}}+\Gamma_{a k}^{i} \frac{\partial \xi^{a}}{\partial x^{j}}+\xi^{a} \frac{\partial \Gamma_{j k}^{i}}{\partial x^{a}}+\left.\Gamma_{j k}^{i}\right|_{m} ^{\sigma} \frac{\partial \xi^{m}}{\partial x^{a}} p_{\sigma}^{a} \\
=\left.\phi_{\alpha \beta}^{\gamma}\right|_{j k} ^{\alpha \beta} p_{\gamma}^{i}+\left.\phi_{\alpha \beta}^{\beta}\right|_{j} ^{\alpha} \delta_{k}^{i}+\left.\phi_{\alpha \beta}^{\alpha}\right|_{k} ^{\beta} \delta_{j}^{i} .
\end{aligned}
$$

Contracting for $i$ and $j$ and observing that $\left(^{(}\right)$

$$
\left.\left.\phi_{\alpha \beta}^{\gamma}\right|_{h k p_{\gamma}^{\alpha \beta}} ^{\alpha \beta} \equiv p_{\gamma}^{h} \frac{\partial}{\partial p_{\alpha}^{h}} \phi_{\alpha \beta}^{\gamma}\right|_{k} ^{\beta}=0
$$

we shall find

$$
\left.\phi_{\alpha \beta}^{\beta}\right|_{k} ^{\alpha}=\frac{1}{N+1} a_{\cdot h k}^{h}
$$

Substitution of (2.23) into (2.20) gives immediately

$$
a_{\cdot j k}^{i}-\frac{\delta_{k}^{i}}{N+1} a_{\cdot h j}^{h}-\frac{\delta_{j}^{i}}{N+1} a_{\cdot h k}^{h}=\left.\phi_{\alpha \beta}^{\gamma}\right|_{j k} ^{\alpha \beta} p_{\gamma \cdot}^{i}
$$

If the last equation be differentiated partially with respect to $p_{\gamma}^{l}$ and the resulting equation be contracted for $i$ and $l$, we have, on observing

$$
\left.\left.\phi_{\alpha \beta}^{p}\right|_{j k l} ^{\alpha \beta \gamma} p_{p}^{l} \equiv p_{p}^{l} \frac{\partial}{\partial p_{\gamma}^{l}} \phi_{\alpha \beta}^{p}\right|_{j k} ^{\alpha \beta \beta}=-\left.K \phi_{\alpha \beta}^{\gamma}\right|_{j k,} ^{\alpha \beta}
$$

because of the generalized homogeneity property of $\phi$, the following relation:

$$
\left.\phi_{\alpha \beta}^{\gamma}\right|_{j k} ^{\alpha \beta}=\frac{1}{N-K}\left(\left.a_{\cdot j k}^{h}\right|_{h} ^{\gamma}-\left.\frac{1}{N+1} a_{\cdot h j}^{h}\right|_{k} ^{\gamma}-\left.\frac{1}{N+1} a_{\cdot h k}^{h}\right|_{j} ^{\gamma}\right)
$$

The functions $\phi$ in (2.21) can now be eliminated by means of (2.24) and (2.26). A reference to $(2.10)$ gives the required conditions for $\xi^{i}(x)$ :

( $\left.{ }^{\circ}\right)$ Using the generalized Euler's equation for a function of the generalized homogeneity property. Cf. Douglas, loc. cit. p. 712, formula (4.8). 


$$
\begin{aligned}
\frac{\partial^{2} \xi^{i}}{\partial x^{i} \partial x^{k}}-\Pi_{j k}^{m} \frac{\partial \xi^{i}}{\partial x^{m}}+ & \Pi_{j m}^{i} \frac{\partial \xi^{m}}{\partial x^{k}}+\Pi_{m k}^{i} \frac{\partial \xi^{m}}{\partial x^{j}} \\
& +\xi^{m} \frac{\partial \Pi_{j k}^{i}}{\partial x^{m}}+\left.\Pi_{j k}^{i}\right|_{l} ^{\sigma} \frac{\partial \xi^{l}}{\partial x^{m}} p_{\sigma}^{m}=\delta_{j}^{i} \psi_{\cdot k}+\delta_{k}^{i} \psi \cdot j
\end{aligned}
$$

where we have placed( $\left.{ }^{7}\right)$

$$
\begin{aligned}
\Pi_{j k}^{i}=\Gamma_{j k}^{i} & -\frac{\delta_{j}^{i}}{N+1} \Gamma_{a k}^{a}-\frac{\delta_{k}^{i}}{N+1} \Gamma_{j a}^{a} \\
& -\frac{p_{\lambda}^{i}}{N-K}\left(\left.\Gamma_{j k}^{a}\right|_{a} ^{\lambda}-\left.\frac{1}{N+1} \Gamma_{a k}^{a}\right|_{i} ^{\lambda}-\left.\frac{1}{N+1} \Gamma_{j a}^{a}\right|_{k} ^{\lambda}\right)
\end{aligned}
$$

and

$$
\psi \cdot j=\frac{1}{N+1} \frac{\partial^{2} \xi^{h}}{\partial x^{h} \partial x^{i}} .
$$

It is to be noted that $\left.\psi_{\cdot j}\right|_{m} ^{\sigma}=0$.

3. The conditions of integrability. To find the conditions of integrability of equations (2.27) we must now introduce the descriptive derivative of a tensor defined in the same way as the covariant derivative of a tensor was defined [cf. (2.12)] provided that the affine connection coefficients $\Gamma_{j k}^{f}$ are replaced by $\Pi_{\mathfrak{k}}^{\mathfrak{k}}$. Designating this new derivation by a subscript preceded by a bar we have particularly

$$
\left.\xi^{i}\right|_{j}=\frac{\partial \xi^{i}}{\partial x^{j}}+\Pi_{j m}^{i} \xi^{m}
$$

and

$$
\begin{aligned}
\left.\xi^{i}\right|_{j k} & =\frac{\partial^{2} \xi^{i}}{\partial x^{i} \partial x^{k}}-\Pi_{j k}^{m} \frac{\partial \xi^{i}}{\partial x^{m}}+\Pi_{j m}^{i} \frac{\partial \xi^{m}}{\partial x^{k}}+\Pi_{m k}^{i} \frac{\partial \xi^{m}}{\partial x^{j}} \\
& +\xi^{m} \frac{\partial^{i} \Pi_{j k}}{\partial x^{m}}+\left.\Pi_{j k}^{i}\right|_{i} ^{\sigma} \frac{\hat{o} \xi^{l}}{\partial x^{m}} p_{\sigma}^{m}-B_{\cdot j k l \xi^{i}}^{i}-\left.\left.\Pi_{j k}^{i}\right|_{l \xi^{\sigma}} ^{\sigma}\right|_{m} p_{\sigma}^{m},
\end{aligned}
$$

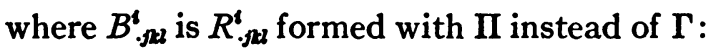

$$
B_{\cdot j k l}^{i}=\frac{\partial \Pi_{j k}^{i}}{\partial x^{l}}-\frac{\partial \Pi_{j l}^{i}}{\partial x^{k}}+\Pi_{a l}^{i} \Pi_{j k}^{a}-\Pi_{a k}^{i} \Pi_{j l}^{a}+\left(\left.\Pi_{m k}^{a} \Pi_{j l}^{i}\right|_{a} ^{b}-\left.\Pi_{m l}^{a} \Pi_{j k}^{i}\right|_{a} ^{a}\right) p_{\sigma}^{m}
$$

By use of (3.2) equations (2.27) may be written as

(7) Cf. Douglas, loc. cit. p. 722, formula (7.8). 


$$
\left.\xi^{i}\right|_{j k}+B_{\cdot j k l \xi^{l}}^{i}+\left.\left.\Pi_{j k}^{i}\right|_{i \xi^{j}}\right|_{\sigma}=\delta_{j}^{i} \psi \cdot k+\delta_{k}^{i} \psi \cdot j,
$$

with $\left.\xi^{l}\right|_{\sigma}=\left.\xi^{l}\right|_{m} p_{\sigma}^{m}$.

For the present necessity and further development it is convenient to utilize the Lie derivative of a geometric object as Davies has shown in the case of affine geometry $\left.{ }^{8}\right)$. First of all, we shall demonstrate that equation (3.4) can simply be expressed in terms of this derivative. In fact, when the coordinate system is changed the law of transformation of $\Pi$ is easily found to be

$$
\frac{\partial \bar{x}^{a}}{\partial x^{j}} \frac{\partial \bar{x}^{b}}{\partial x^{k}} \bar{\Pi}_{a b}^{i}=\frac{\partial \bar{x}^{i}}{\partial x^{a}} \Pi_{j k}^{a}-\frac{\partial^{2} \bar{x}^{i}}{\partial x^{i} \partial x^{k}}+\frac{\partial \bar{x}^{i}}{\partial x^{j}} \theta_{k}+\frac{\partial \bar{x}^{i}}{\partial x^{k}} \theta_{j}
$$

where

$$
\theta_{j}=\frac{1}{N+1} \frac{\partial \log \Delta}{\partial x^{i}}
$$

$\Delta=|(\partial \bar{x} / \partial x)|$ denoting the Jacobian of the transformation $\left({ }^{(}\right)$.

Substituting (2.4) into (3.5) and neglecting, as before, higher powers of $\delta t$ than the first, we are led to the relation

$$
\begin{aligned}
\bar{\Pi}_{j k}^{i}(\bar{x}, \bar{p})=\Pi_{j k}^{i}+\left\{\Pi_{j k}^{m} \frac{\partial \xi^{i}}{\partial x^{m}}\right. & -\Pi_{j m}^{i} \frac{\partial \xi^{m}}{\partial x^{k}}-\Pi_{m k}^{i} \frac{\partial \xi^{m}}{\partial x^{j}} \\
& \left.+\frac{1}{N+1}\left(\delta_{j}^{i} \psi \cdot k+\delta_{k}^{i} \psi \cdot j\right)-\frac{\partial^{2} \xi^{i}}{\partial x^{j} \partial x^{k}}\right\} \delta t .
\end{aligned}
$$

Similarly, we obtain

$$
\Pi_{j k}^{i}(\bar{x}, \bar{p})=\Pi_{j k}^{i}+\left(\frac{\partial \Pi_{j k}^{i}}{\partial x^{m}} \xi^{m}+\left.\Pi_{j k}^{i}\right|_{m} ^{\sigma} \frac{\partial \xi^{m}}{\partial x^{l}} p_{\sigma}^{l}\right) \delta t,
$$

whence follows the Lie derivative of $\Pi_{j k}^{t}$ :

$$
\begin{aligned}
\Delta\left(\Pi_{j k}^{i}\right) \equiv & \lim _{\delta t \rightarrow 0} \frac{\Pi_{j k}^{i}(\bar{x}, \bar{p})-\bar{\Pi}_{j k}^{i}(\bar{x}, \bar{p})}{\delta t} \\
= & \frac{\partial^{2} \xi^{i}}{\partial x^{i} \partial x^{k}}-\Pi_{j k}^{m} \frac{\partial \xi^{i}}{\partial x^{m}}+\Pi_{j m}^{i} \frac{\partial \xi^{m}}{\partial x^{k}}+\Pi_{m k}^{i} \frac{\partial \xi^{m}}{\partial x^{j}}+\xi^{m} \frac{\partial \Pi_{j k}^{i}}{\partial x^{m}} \\
& +\left.\Pi_{j k}^{i}\right|_{m} ^{\cdot} \frac{\partial \xi^{m}}{\partial x^{l}} p_{o}^{l}-\frac{1}{N+1}\left(\delta_{j \psi \cdot k}^{i}+\delta_{k}^{i} \psi \cdot j\right) .
\end{aligned}
$$

(8) Cf. Davies, loc. cit. p. 103, formula (2.6).

(9) Several signs in formula (8.9) of Douglas' paper (loc. cit.) should be changed. Similar corrections for formulas (5.5), (8.6) and (8.11). Also see Knebelman, loc. cit. formulas (1.13), (4.3) (the latter lacks the term $\left.\partial^{2} x^{i} /\left(\partial x^{\beta} \partial x^{\gamma}\right)\right)$ and Davies, loc. cit. formula (1.3). 
Therefore the conditions for an infinitesimal descriptive collineation may be written simply

$$
\Delta\left(\Pi_{j k}^{i}\right)=0 .
$$

As in the case of affine geometry it is readily shown that if $\xi^{i}(x)$ determines a descriptive collineation of the space, the Lie derivative is permutable with the two operators denoted respectively by $\left.\right|_{j}$ and $\left.\right|_{m} ^{\sigma}$.

The following permutation formulas for the operations just quoted are easily obtainable:

$$
\begin{aligned}
\left.X^{i}\right|_{j k}-\left.X^{i}\right|_{k j} & =\left(\delta_{n}^{i} X^{m}-\left.X^{i}\right|_{n} ^{p} p_{\rho}^{m}\right) B_{\cdot m j k}^{n}, \\
\left.X^{i}{ }_{j}\right|_{k} ^{\alpha}-\left.\left.X^{i}\right|_{k} ^{\alpha}\right|_{j} & =\left.\left(\delta_{n}^{i} X^{m}-\left.X^{i}\right|_{n} ^{\rho} p_{\rho}^{m}\right) \Pi_{m j}^{n}\right|_{k} ^{\alpha},
\end{aligned}
$$

with obvious extensions to any tensor. We also need the generalization of the Bianchi identity in the form $\left({ }^{10}\right)$

$$
\left.B_{. j m k}^{\imath}\right|_{\imath}+\left.B_{. j k l}^{\imath}\right|_{m}+\left.B_{. g l m}^{\imath}\right|_{k}
$$

Let us now consider the conditions of integrability of (3.4). For this purpose we have

$$
\begin{aligned}
& \left.\xi^{i}\right|_{j k l}=\left.\delta_{j}^{i} \psi \cdot k\right|_{l}+\left.\delta_{k}^{i} \psi \cdot j\right|_{l}+\left.\Pi_{j k}^{i}\right|_{r} ^{\sigma} p_{\sigma}^{m} \dot{B}_{\cdot m l a}^{r} \xi^{a} \\
& -\left.\left.\left.\Pi_{j k}^{i}\right|_{h} ^{\sigma}\right|_{l \xi^{h}}\right|_{\sigma}+\left.\left.\left.\Pi_{j k}^{i}\right|_{r} ^{\sigma} \Pi_{m l}^{r}\right|_{\sigma} ^{\rho} \xi^{\sigma}\right|_{q} p_{\sigma}^{m} p_{\rho}^{q} \\
& -\left.\xi^{h}\right|_{l} B_{\cdot j k m}^{i}-\left.\xi^{h} B_{\cdot j k h}^{i}\right|_{l} \text {. }
\end{aligned}
$$

If from this equation we subtract the one obtained from it by interchange of $k$ and $l$, make use of the fact that

$$
\left.\left.\xi^{i}\right|_{j}\right|_{h} ^{\alpha}=\left.\xi^{r} \Pi_{j r}^{i}\right|_{h} ^{\alpha}
$$

of the equation

$$
\left.\xi^{i}\right|_{j k l}-\left.\xi^{i}\right|_{j l k}=-\left.\left.\xi^{i}\right|_{j}\right|_{h} ^{\alpha} B_{\cdot m k l}^{h} p_{\alpha}^{m}+\left.B_{\cdot h k l}^{i} \xi^{h}\right|_{i}-\left.B_{\cdot j k l}^{h} \xi^{i}\right|_{h,}
$$

and of the relation

$$
\left.\Pi_{j k}^{i}\right|_{m p} ^{\sigma} p_{p}^{m}=0 \quad \text { (for all } \rho, \sigma \text { and } i, j, k \text { ), }
$$

the last being deducible from the definition of $\Pi$ and an equation given by Douglas (loc. cit. formula (5.8)):

$$
\left.\left.\Gamma_{j k}^{i}\right|_{m p_{\rho}^{m}} ^{\sigma}=0 \quad \text { (for all } \rho, \sigma \text { and } i, j, k\right),
$$

we shall have as the result

(10) Davies, loc. cit. p. 102, formula (1.9). 


$$
\begin{aligned}
\left.\xi^{m} B_{\cdot j k l}^{i}\right|_{m}+\left.\left.B_{\cdot j k l}^{i}\right|_{h} \xi^{\sigma} \xi^{h}\right|_{\sigma}-\left.\xi^{i}\right|_{h} B_{\cdot j k l}^{h}+\left.\xi^{h}\right|_{j} B_{\cdot h k l}^{i} \\
+\left.\xi^{m}\right|_{k} B_{\cdot{ }_{j m l}}^{i}+\left.\xi^{m}\right|_{l} B_{\cdot j k m}^{i}=\left.\delta_{k l \cdot j}^{i}\right|_{l}-\left.\delta_{l}^{i} \psi \cdot j\right|_{k .}
\end{aligned}
$$

By contraction in (3.18) for $i$ and $l$ we obtain

$$
\left.\xi^{m} B_{\cdot j k a}^{a}\right|_{m}+\left.\left.B_{\cdot j k a}^{a}\right|_{h \xi^{\sigma}} ^{\sigma}\right|_{\sigma}+\left.\xi^{h}\right|_{j} B_{\cdot h k a}^{a}+\left.\xi^{m}\right|_{k} B_{\cdot j m a}^{a}=-\left.(N-1) \psi_{\cdot j}\right|_{k}
$$

and, similarly, by contraction in (3.18) for $i$ and $k$,

$$
\left.\xi^{m} B_{\cdot{ }_{j a l}}^{a}\right|_{m}+\left.\left.B_{\cdot{ }_{j a l}}^{a}\right|_{h \xi^{a}} ^{\sigma}\right|_{\sigma}+\left.\xi^{h}\right|_{j} B_{\cdot h a l}^{a}+\left.\xi^{m}\right|_{l} B{ }_{\cdot j a m}^{a}=\left.(N-1) \psi_{\cdot i}\right|_{l \cdot}
$$

On substituting (3.19) and (3.20) into (3.18) and reducing, we obtain the conditions of integrability:

$$
\begin{aligned}
\left.\xi^{m} W_{\cdot j k l}^{i}\right|_{m}+\left.\left.\xi^{h}\right|_{m} W_{\cdot j k l}^{i}\right|_{h p_{\sigma}^{m}} ^{\sigma}+\left.\xi^{h}\right|_{j} W_{\cdot h k l}^{i} & \\
& -\left.\xi^{i}\right|_{h} W_{\cdot j k l}^{h}+\left.\xi^{m}\right|_{k} W_{\cdot j m l}^{i}+\left.\xi^{m}\right|_{l} W_{\cdot j k m}^{i}=0
\end{aligned}
$$

or, in terms of the Lie derivatives,

$$
\Delta\left(W_{\cdot j k l}^{i}\right)=0,
$$

where $W^{t}$.pk are the components of the descriptive curvature tensor (cf. Douglas, loc. cit. p. 727):

$$
W_{\cdot j k l}^{i}=B_{\cdot j k l}^{i}-\frac{\delta_{l}^{i}}{N-1} B_{\cdot j k a}^{a}-\frac{\delta_{k}^{i}}{N-1} B_{\cdot j a l \cdot}^{a}
$$

The conditions of integrability of (3.4) with respect to $p$ are obtainable by partial differentiation of (3.4). But we find it better to treat it directly from (2.27), because the partial derivatives of $\xi^{i}, \partial \xi^{i} / \partial x^{i}, \partial^{2} \xi^{i} /\left(\partial x^{j} \partial x^{k}\right)$ with respect to the process $\left.\right|_{m} ^{\sigma}$ all vanish. The resulting equation is as follows:

$$
\begin{aligned}
\left.\Pi_{j h}^{i}\right|_{n} ^{\rho} \frac{\partial \xi^{h}}{\partial x^{k}}+\left.\Pi_{h k}^{i}\right|_{n} ^{\rho} & \frac{\partial \xi^{h}}{\partial x^{j}}-\left.\Pi_{j k}^{h}\right|_{n} ^{\rho} \frac{\partial \xi^{i}}{\partial x^{h}} \\
& +\left.\xi^{h} \frac{\partial}{\partial x^{h}} \Pi_{j k}^{i}\right|_{n} ^{\rho}+\left.\Pi_{j k}^{i}\right|_{h} ^{\rho} \frac{\partial \xi^{h}}{\partial x^{n}}+\left.\Pi_{j k}^{i}\right|_{n h} ^{\rho \sigma} \frac{\partial \xi^{h}}{\partial x^{m}} p_{\sigma}^{m}=0
\end{aligned}
$$

and it is easily seen that (3.24) may be written as

$$
\Delta\left(\left.\Pi_{j k}^{i}\right|_{n} ^{p}\right)=0 .
$$

The tensor form of the last equation follows immediately from (3.1) as: well as from the fact that $\left.\pi_{f k}^{j}\right|_{n} ^{p}$ is a tensor covariant in $j, k, n$ and contravariant in $i$. Thus we obtain as further conditions of integrability

$$
\begin{aligned}
\left.\left.\xi^{h} \Pi_{j k}^{i}\right|_{n} ^{\rho}\right|_{h}+\left.\left.\xi^{h}\right|_{m} \Pi_{j k}^{i}\right|_{n h} ^{\rho \sigma} p_{\sigma}^{m} & +\left.\left.\xi^{h}\right|_{j} \Pi_{h k}^{i}\right|_{n} ^{\rho} \\
& -\left.\left.\xi^{i}\right|_{h} \Pi_{j k}^{h}\right|_{n} ^{\rho}+\left.\left.\xi^{h}\right|_{k} \Pi_{j h}^{i}\right|_{n} ^{\rho}+\left.\left.\xi^{h}\right|_{n} \Pi_{j k}^{i}\right|_{h} ^{\rho}=0 .
\end{aligned}
$$


Because of (3.16) and its derivation with respect to $p$ the following relations are valid:

$$
\begin{aligned}
& \left.p_{\sigma}^{n 1} \Pi_{j k}^{i}\right|_{n 1} ^{p 1}=0 \text {, } \\
& \left.p_{\sigma}^{n_{2} 2} \Pi_{j k}^{i}\right|_{n 1 n_{2}} ^{p_{1} p_{2}}=-\left.\delta_{\sigma}^{\rho_{1}} \Pi_{j k}^{i}\right|_{n 1} ^{\rho_{2}}
\end{aligned}
$$

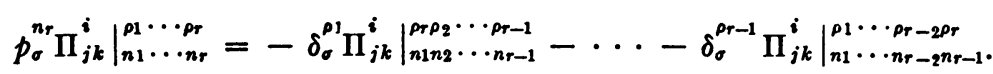

According to these equations and the permutability property of the $\Delta$ operator with $\left.\right|_{j}$ and $\left.\right|_{m} ^{\sigma}$ we may conclude as in the case of affine collineations treated by Davies that if

$$
\Delta\left(\left.W_{\cdot j k l}^{i}\right|_{m_{1} m_{2}} \cdots m_{s}\right)=0 \quad(s=1,2, \cdots)
$$

and

$$
\Delta\left(\left.\left.\Pi_{j k}^{i}\right|_{n 1} ^{\rho_{1} \cdots p_{r r}}\right|_{l_{1} l_{2} \cdots l_{p}}\right)=0 \quad \quad(r=1,2, \cdots)
$$

form a finite number of conditions which are compatible, then the three sets (3.4), (3.27) and (3.28) form a completely integrable system of equations, and the space of $K$-spreads admits descriptive collineations. This generalizes the result of Knebelman on projective collineations in generalized spaces.

4. Descriptive flatness of a space of $K$-spreads. We come now to establish some theorems as immediate consequences of equations (3.4) and their conditions of integrability. Consider, in fact, a solution $\xi^{i}$ of equations (2.27) which are equivalent to (3.4). By a suitable transformation of coordinates we can normalize the vector $\xi^{i}$ so that its components are $\delta_{1}^{i}$. Since equations (3.4) are in tensor form, they remain in the same form and so do (2.27), the latter reducing in this coordinate system to

$$
\frac{\partial \Pi_{j k}^{i}}{\partial x^{1}}=0 .
$$

The finite transformations generated by the above vector are

$$
\bar{x}^{i}=x^{i}+a \delta_{1}^{i},
$$

for which the jacobian $\Delta=1$, so that equations (3.5) give $\bar{\Pi}_{j k}^{i}=\Pi_{j k}^{i}$. That is, the transformations (4.2) carry $K$-spreads into $K$-spreads. Thus we have proved the theorem:

$A$ necessary and sufficient condition that a vector $\xi^{i}$ must satisfy in order to define an infinitesimal descriptive collineation is that it be a solution of (2.27).

From (4.1) follows the theorem:

The most general connection in a space of $K$-spreads admitting an infinitesimal descriptive collineation may be obtained by choosing, for the coefficients of 
descriptive connection, functions of $N-1$ of the coordinates $x$ having the generalized homogeneity property of the second degree in $p_{\sigma}^{l}$.

From (4.2) we have the theorem:

$A$ manifold admitting an infinitesimal descriptive collineation admits the finite continuous group $G_{1}$ of descriptive collineations.

In the next section it is shown that this theorem may be generalized to an $r$-parameter group $G_{r}$ of descriptive collineations.

Returning to (3.4) we see that if they are to be completely integrable, equations (3.26) and (3.21) must be identities in the $N^{2}+N$ quantities $\xi^{i} \mid$, and $\xi^{i}$. If we write these equations in the form

$$
\begin{aligned}
& \left.\left.\xi^{h} \Pi_{j k}^{i}\right|_{n} ^{p}\right|_{h}+\left.\xi^{h}\right|_{m}\left(\left.\Pi_{j k}^{i}\right|_{n h} ^{\rho \sigma} p_{\sigma}^{m}-\left.\delta_{h}^{i} \Pi_{j k}^{m}\right|_{n} ^{p}+\left.\delta_{j}^{m} \Pi_{h k}^{i}\right|_{n} ^{p}\right. \\
& \left.+\left.\delta_{k}^{m} \Pi_{j h}^{i}\right|_{n} ^{p}+\left.\delta_{n}^{m} \Pi_{j k}^{i}\right|_{h} ^{p}\right)=0, \\
& \left.\xi^{h} W_{\cdot j k l}^{i}\right|_{h}+\left.\xi^{h}\right|_{m}\left(\left.W_{\cdot j k l}^{i}\right|_{h p_{\sigma}} ^{m}-\delta_{h}^{i} W_{\cdot j k l}^{m}+\delta_{j}^{m} W_{\cdot h k l}^{i}\right. \\
& \left.+\delta_{k}^{m} W_{\cdot j h l}^{i}+\delta_{l}^{m} W_{\cdot j k h}^{i}\right)=0 \text {, }
\end{aligned}
$$

it follows that

$$
\left.\left.\Pi_{j k}^{i}\right|_{n} ^{p}\right|_{h}=0,\left.\quad W_{\cdot j k l}^{i}\right|_{h}=0
$$

and each parenthesis in (4.3) and (4.4) must vanish. When these last two quantities are contracted for $m$ and $h$ we obtain

$$
\left.\Pi_{j k}^{i}\right|_{n} ^{p}=0, \quad W_{\cdot j k l}^{i}=0 .
$$

Douglas (loc. cit. p. 728) has shown that these are necessary and sufficient conditions that a space of $K$-spreads be transformable into

$$
\frac{\partial^{2} x^{i}}{\partial u^{\alpha} \partial u^{\beta}}=0
$$

or, what is equivalent,

$$
x^{i}=A_{\alpha}^{i} u^{\alpha}+C^{i}
$$

by arbitrary changes of coordinates and parameters. Hence:

A necessary and sufficient condition that a descriptive connection must satisfy in order to admit $N^{2}+2 N$ linearly independent descriptive collineations is that it be descriptively flat.

5. Group property of descriptive collineations. The last theorem in $\$ 4$ furnishes a characteristic property of descriptively flat spaces of $K$-spreads. It is for no other reason than this that the isomorphic transformations are called descriptive collineations. Another fact which affirms the nomination may be formulated more concretely as follows:

If a space of $K$-spreads admits $r$ linearly independent infinitesimal descrip- 
tive collineations it admits the $r$-parameter group $G_{r}$ generated by them.

For let $\xi^{i}$ and $\eta^{i}$ be any two of the $r$ linearly independent solutions that equations (3.4) are assumed to admit and let $X \equiv \xi^{i} \partial / \partial x^{i}, Y \equiv \eta^{i} \partial / \partial x^{i}$ be the corresponding generators. In order to prove the above theorem it is necessary and sufficient to show that the Poisson operator $(X, Y)$ is expressible linearly with constant coefficients in terms of the $r$ generators. Let $(X, Y)=\lambda i \partial / \partial x^{i}$. Then we have

$$
\lambda^{i}=\xi^{k} \partial \eta^{i} / \partial x^{k}-\eta^{k} \partial \xi^{i} / \partial x^{k}
$$

or better

$$
\lambda^{i}=\left.\xi^{m} \eta^{i}\right|_{m}-\left.\eta^{m} \xi^{i}\right|_{m} .
$$

By descriptive differentiation we obtain

$$
\left.\lambda^{i}\right|_{j}=\left.\left.\xi^{h}\right|_{j} \eta^{i}\right|_{h}-\left.\left.\eta^{h}\right|_{j \xi^{i}}\right|_{h}+\left.\xi^{h} \eta^{i}\right|_{h i}-\left.\eta^{h} \xi^{i}\right|_{h j}
$$

and since $\xi^{i}$ and $\eta^{i}$ satisfy (3.4), we get by means of these equations

$$
\begin{gathered}
\left.\lambda^{i}\right|_{j}=\left.\left.\xi^{h}\right|_{j \eta^{i}}\right|_{h}-\left.\left.\eta^{h}\right|_{j \xi^{i}}\right|_{h}+\xi^{h} \eta^{l} B_{\cdot j l h}^{i}-\left.\Pi_{j h}^{i}\right|_{l} ^{\sigma}\left(\left.\xi^{h} \eta^{l}\right|_{m}-\left.\eta^{h} \xi^{l}\right|_{m}\right) p_{\sigma}^{m} \\
+\xi^{i} \phi \cdot j-\eta^{i} \psi \cdot j+\delta_{j}^{i}\left(\xi^{h} \phi \cdot h-\eta^{h} \psi \cdot h\right),
\end{gathered}
$$

where in accordance with (2.29)

$$
\psi_{\cdot j}=\frac{1}{N+1} \frac{\partial^{2} \xi^{h}}{\partial x^{h} \partial x^{j}}, \quad \phi \cdot j=\frac{1}{N+1} \frac{\partial^{2} \eta^{h}}{\partial x^{h} \partial x^{j}} .
$$

When (5.2) are differentiated descriptively and second derivatives of $\xi^{i}$ and $\eta^{i}$ are eliminated by means of (3.4) we obtain

$$
\begin{aligned}
& \left.\lambda^{i}\right|_{j k}=B_{\cdot j k l}^{h}\left(\left.\xi^{i}\right|_{h} \eta^{l}-\left.\eta^{i}\right|_{h} \xi^{l}\right)+B_{\cdot h k l}^{i}\left(\left.\xi^{l} \eta^{h}\right|_{j}-\left.\eta^{l} \xi^{h}\right|_{j}\right) \\
& +B^{i}{ }_{j l h}\left(\left.\xi^{h}\right|_{k \eta^{l}}-\left.\eta^{h}\right|_{k \xi^{l}}\right)+\left.\xi^{h} \eta^{l} B^{i} \cdot{ }_{j l h}\right|_{k} \\
& +\left.\Pi_{j k}^{h}\right|_{l} ^{\sigma}\left(\left.\left.\xi^{i}\right|_{h} \eta^{l}\right|_{\sigma}-\left.\left.\eta^{i}\right|_{h} \xi^{l}\right|_{\sigma}\right) \\
& +\left.\Pi_{h k}^{i}\right|_{l} ^{\sigma}\left(\left.\left.\xi^{l}\right|_{\sigma} \eta^{h}\right|_{j}-\left.\left.\eta^{l}\right|_{\sigma} \xi^{h}\right|_{j}\right) \\
& +\left.\left.\Pi_{j h}^{i}\right|_{l} ^{\sigma}\right|_{k}\left(\left.\xi^{l}\right|_{\sigma} \eta^{h}-\left.\eta^{l}\right|_{\sigma} \xi^{h}\right) \\
& +\left.\Pi_{j h}^{i}\right|_{l^{\sigma} B} ^{\sigma}{ }^{l}{ }_{m k r} p_{\sigma}^{m}\left(\xi^{h} \eta^{r}-\eta^{h} \xi^{r}\right) \\
& +\left.\left.\Pi_{j h}^{i}\right|_{l} ^{\sigma} \Pi_{m k}^{l}\right|_{r} ^{p} p_{\rho}^{i} p_{\sigma}^{m}\left(\left.\xi \eta^{h}\right|_{\sigma}-\left.\eta^{h} \xi^{r}\right|_{\sigma}\right) \\
& +\delta_{k}^{i}\left(\left.\xi^{h}\right|_{j} \phi \cdot h-\left.\eta^{h}\right|_{j} \psi \cdot h\right) \\
& +\left.\xi^{i} \phi \cdot j\right|_{k}-\left.\eta^{i} \psi \cdot j\right|_{k} \\
& +\delta_{j}^{i}\left(\left.\xi^{h}\right|_{k} \phi \cdot h-\left.\eta^{h}\right|_{k} \psi_{\cdot h}+\left.\xi^{h} \phi \cdot h\right|_{k}-\left.\eta^{h} \psi_{\cdot h}\right|_{k}\right) \\
& +\left.\Pi_{j h}^{i}\right|_{l} ^{\sigma}\left(\left.\left.\xi^{l}\right|_{\sigma} \eta^{h}\right|_{k}-\left.\left.\eta^{l}\right|_{\sigma} \xi^{h}\right|_{k}\right) \text {. }
\end{aligned}
$$


In deriving (5.3) use is made of (3.16) and

$$
p_{\sigma}^{m} \psi \cdot m=0, \quad p_{\sigma}^{m} \phi \cdot m=0 .
$$

From (5.1) and (5.2) we have

$$
B_{\cdot j k l}^{i} \xi^{l}=B_{\cdot j k l}^{i}\left(\left.\xi^{m} \eta^{l}\right|_{m}-\left.\eta^{m} \xi^{l}\right|_{m}\right)
$$

and

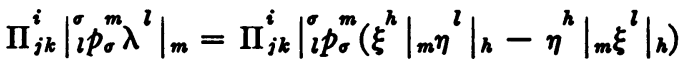

$$
\begin{aligned}
& +\left.\Pi_{j k}^{i}\right|_{l p_{\sigma}^{\infty} \xi^{m} \eta^{r} B} ^{l} B_{m r h} \\
& -\left.\left.\Pi_{j k}^{i}\right|_{i} ^{\sigma} \Pi_{m h}^{l}\right|_{r} ^{\rho}\left(\left.\xi^{h} \eta^{r}\right|_{\odot}-\left.\eta^{h} \xi^{r}\right|_{\varepsilon}\right) p_{\sigma}^{m} p_{\rho}^{\dot{0}} .
\end{aligned}
$$

Adding these two expressions to (5.3) and using (3.13), (3.18) and

$$
\left.B_{\cdot j k h}^{i}\right|_{m} ^{\rho}=\left.\left.\Pi_{j k}^{i}\right|_{m} ^{\rho}\right|_{h}-\left.\left.\Pi_{j h}^{i}\right|_{m} ^{\rho}\right|_{k}+\left(\left.\left.\Pi_{j h}^{i}\right|_{l} ^{\sigma} \Pi_{r k}^{l}\right|_{m} ^{\rho}-\left.\left.\Pi_{j k}^{i}\right|_{l} ^{\sigma} \Pi_{r h}^{l}\right|_{m} ^{\rho}\right) p_{\sigma}^{r},
$$

we find

$$
\begin{aligned}
\left.\lambda^{i}\right|_{j k}+B_{\cdot j k \lambda}^{i} \lambda^{l} & +\left.\Pi_{j k}^{i}\right|_{\left.l p_{\sigma} \lambda^{2} \lambda^{l}\right|_{m}} \\
= & \delta_{j}^{i}\left(\left.\xi^{h} \phi \cdot h\right|_{k}-\left.\eta^{h} \psi \cdot h\right|_{k}+\left.\xi^{h}\right|_{k} \phi \cdot h-\left.\eta^{h}\right|_{k} \psi \cdot h\right) \\
& +\delta_{h}^{i}\left(\left.\xi^{h} \phi \cdot j\right|_{h}-\left.\eta^{h} \psi \cdot i\right|_{h}+\left.\xi^{h}\right|_{j} \phi \cdot h-\left.\eta^{h}\right|_{j} \psi \cdot h\right) .
\end{aligned}
$$

A reference to the definition of $\psi$ and $\phi$ shows

$$
\left.\psi \cdot j\right|_{h}=\left.\psi \cdot h\right|_{j},\left.\quad \phi \cdot j\right|_{h}=\left.\phi \cdot h\right|_{j \cdot}
$$

Hence, on putting

$$
x=\xi^{r} \phi \cdot r-\eta^{r} \psi_{\cdot r}=\frac{1}{N+1} \frac{\partial \lambda^{h}}{\partial x^{h}},
$$

we can write (5.8) in the form

$$
\left.\lambda^{i}\right|_{j k}+B_{\cdot j k l}^{i} \lambda^{l}+\left.\Pi_{j k}^{i}\right|_{\left.l p_{\sigma}{ }^{m} \lambda^{l}\right|_{m}=\delta_{j \cdot k}^{i}+\delta_{k}^{i} x \cdot j .}
$$

That $\lrcorner, \lambda^{i}$ is therefore a solution of (3.4), which is homogeneous of the first degree; this implies that if $\xi^{i}(\alpha)(\alpha=1,2, \cdots, r)$ are the $r$ linearly independent solutions of (3.4), $\lambda^{i}=a^{\alpha} \xi^{i}(\alpha)$, where $a^{\alpha}$ are a set of constants. Hence if $X_{\alpha}=\xi^{i}(\alpha) \partial / \partial x^{i}$ we have $\left(X_{\alpha}, X_{\beta}\right) f=c_{\alpha \beta}^{\gamma} X_{\gamma} f, c_{\alpha \beta}^{\gamma}$ being the constants of composition of the group.

National Chekiang University,

HaNGChow, China 